\title{
Readout methods and devices for Josephson-junction-based solid-state qubits.
}

\author{
G. Johansson, L. Tornberg, V.S. Shumeiko and G. Wendin \\ Department of Microtechnology and Nanoscience - MC2, \\ Chalmers University of Technology, \\ SE-41296 Gothenburg, Sweden
}

(Dated: May 27, 2021)

\begin{abstract}
We discuss the current situation concerning measurement and readout of Josephson-junction based qubits. In particular we focus attention of dispersive low-dissipation techniques involving reflection of radiation from an oscillator circuit coupled to a qubit, allowing single-shot determination of the state of the qubit. In particular we develop a formalism describing a charge qubit read out by measuring its effective (quantum) capacitance. To exemplify, we also give explicit formulas for the readout time.
\end{abstract}

\section{INTRODUCTION}

Nanotechnology is considered promising for fabrication of scalable solid-state electronics for quantum computers ${ }^{1,2.3}$. However, progress towards solid-state quantum computing will critically depend on the development of measurement schemes and readout devices that, on demand, can determine the state of individual qubits in a fraction of the coherence time, but which otherwise do not disturb the qubit system. In quantum optics, efficient measurement techniques have been developed during the last thirty years based on laser-atom interactions and recently implemented in e.g. ion traps $4.5,6.7 .8 .9$.

The corresponding work for solid state systems effectively started only about ten years ago, and is currently exploring various paths. A problem is that there is no general device for operation and readout, like a laser, but rather a multitude of implementations of measurements of charge, spin, magnetic flux and charge current that must be adapted to the specific qubits to be studied. Therefore, the qubit readout technology must be developed in intimate connection with the qubits for characterization and control of coherence properties. This is a painstakingly slow process which, however, cannot be circumvented, because it is essential in many respects. In particular, it is an important tool for determining the coherence properties of the qubits. Moreover, the technology not only concerns qubit readout devices, but also involves quantum oscillators for storing and transmitting information and for coupling qubits.

Interestingly enough, quantum-optical methods are now being applied to solid-state qubit systems, using microwaves for operating and reading out qubits, and oscillator circuits and transmission lines for coupling qubits, introducing cavity-QED in solid-state systems ${ }^{10.11 .12}$. This may turn out to be a major road on the Road Map for quantum coherent systems ("quantum computers" $\stackrel{13.14}{\underline{1}}$, and will be at the focus of the present paper. In particular we will describe some practical schemes for reflecting microwaves from an oscillator circuit, the phase shift measuring the changes in charge $\frac{15.16}{5}$ or magnetic flux ${ }^{17.18 .19 .20 .21}$ induced by a qubit, allowing to distinguish between the different states $|0\rangle$ and $|1\rangle$ of the qubit.

\section{MEASUREMENT OF QUANTUM INFORMATION AND QUBIT READOUT}

\section{A. Introduction}

The ultimate objective of a qubit readout device is to distinguish the eigenstates of a qubit in a single measurement "without destroying the qubit", a so called "single-shot" quantum non-demolition (QND) projective measurement. This objective is essential for several reasons: state preparation for computation, readout for error correction during the calculation, and readout of results at the end of the calculation. Strictly speaking, the QND property is only needed if the qubit must be left in an eigenstate after the readout. In a broader sense, readout of a specific qubit must of course not demolish other qubits in the system.

Note that one cannot "read out the state of a qubit" in a single measurement - this is prohibited by quantum mechanics. The effect of a single ideal measurement on a qubit in a general superposition $a|0\rangle+b|1\rangle$ is to leave the qubit in one of the states $|0\rangle$ or $|1\rangle$, which carries no information about the amplitudes $a$ or $b$. It takes repeated measurements on a large number of replicas of the quantum state to characterize the state of the qubit - "quantum tomography". This is the procedure to collect the statistics for expectation values.

The measurement connects the qubit with the open system of the detector, which collapses the combined system of qubit and measurement device to one of its common eigenstates. If the coupling between the qubit and the detector 
is weak, the eigenstates are approximately those of the qubit. In general however, one must consider the eigenstates of the total qubit-detector system and manipulate gate voltages and fluxes such that the readout measurement is performed in a convenient energy eigenbasis (see e.g. Refs. 22 and 23 ).

\section{B. Survey of readout methods for JJ-based qubits}

Here we will provide a brief recapitulation of the "history" of readout of Josephson-junction (JJ) based quantum circuits and qubits. For an extensive discussion of JJ-based qubit circuits, see the recent review by Wendin and Shumeiko ${ }^{3}$.

Figure 1 shows general designs for the charge and flux qubits and with oscillator-type readout circuits. The Single Cooper-pair Box (SCB) (similar to Fig. 1, left) is described by the Hamiltonian

$$
\hat{H}=E_{C}\left(n-n_{g}\right)^{2}-E_{J} \cos \phi
$$

and the rf-SQUID (similar to the flux qubit in Fig. 1, right; see Section VI) by the Hamiltonian

$$
\hat{H}=E_{C} n^{2}-E_{J} \cos \phi+E_{L} \frac{\left(\phi-\phi_{e}\right)^{2}}{2} ;
$$

where $E_{C}$ is the charging energy of the SCB island, $E_{J}$ is the Josephson energy due to Cooper-pair tunneling between the superconducting electrode and island across the JJ, and $E_{L}$ is the inductive energy of the superconducting loop. $n$ and $\phi$ are operators for the induced charge and the phase of the (effective) Josephson junction, and $n_{g}$ is the induced charge controlled by the external bias voltage $V_{g}$. Both qubits are represented by the generic 2-level Hamiltonian

$$
\hat{H}=-\frac{1}{2}\left(\epsilon \sigma_{z}+\Delta \sigma_{x}\right)
$$

where $\sigma_{z}$ and $\sigma_{x}$ are the usual Pauli matrices.
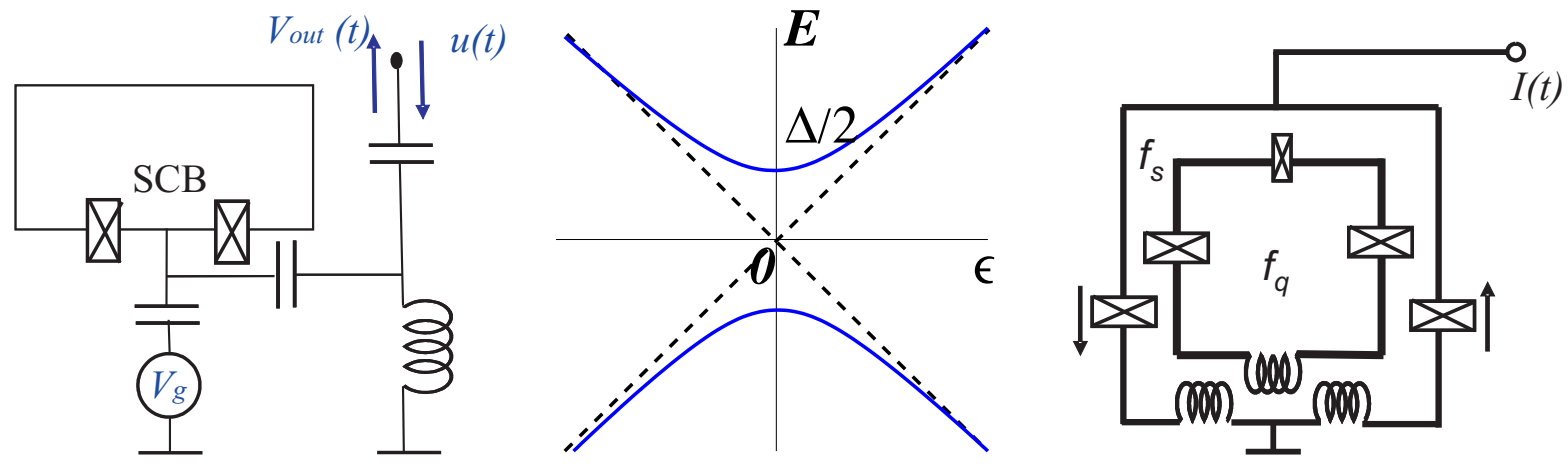

FIG. 1: Circuit diagrams and 2-level energy spectrum of two basic JJ-qubit designs: the SCB charge qubit with LC-oscillator readout (left), and persistent-current flux qubit with SQUID oscillator readout (right). For the charge qubit, the control variable $\epsilon$ on the horizontal axis of the energy spectrum (middle) represents the external gate voltage (induced charge), and the splitting is given by the Josephson tunneling energy mixing the charge states. For the flux qubit, the variable $\epsilon$ represents the external magnetic flux. In both cases, the energy of the qubit can be "tuned" and the working point controlled. Away from the origin (asymptotically) the levels represent pure charge states (zero $|0\rangle$ or one $|1\rangle$ Cooper pair on the SCB island) or pure flux states (left $|0\rangle$ or right $|1\rangle$ rotating currents in the SQUID ring).

In the original experiment of Nakamura et al. ${ }^{24}$, demonstrating coherent oscillation of the charge qubit 2-level system (Fig. 1, left), the readout was implemented simply by a control dc-pulse on the charge gate, moving the working point far away from the origin so that the upper $|1\rangle$ level ended up above the gap edge of a superconducting lead connected to the SCB island via a tunnel junction. As a result, a Cooper pair on the upper level $|1\rangle$ would immediately decay into the external lead as two quasiparticles, creating a normal electron current. Repeating the measurement at a high rate created a detectable current proportional to the occupation of the upper state $|1\rangle$, revealing the oscillations. Since the SCB is permanently connected to the environment via a tunnel junction, it seemed plausible at the time that this might be the reason for the short coherence time, $\sim 2-3 \mathrm{~ns}$.

This focussed the interest on more advanced readout devices. A remedy could be to use a charge measuring device that was only capacitively coupled to the SCB island and could be turned on and off by an external voltage 
pulse. Delsing and coworkers ${ }^{25}$ therefore developed an rf-SET (radio frequency single-electron transistor) readout ${ }^{26}$ for the charge qubit, and successfully detected free oscillations and studied the detailed behaviour of relaxation and dephasing ${ }^{25}$. The result showed that the coherence time was confined to below $10 \mathrm{~ns}$ and seemed limited by relaxation effects. Moreover, subsequent experiments by the NEC group 27 , implementing more advanced readout concepts, storing the emitted pair of quasiparticles on a superconducting island, and reading the charge with a superconducting SET, made no big change. All in all, the status seems to be that the coherence time of the circuit is severely limited by intrinsic charge fluctuation processes (noise) in the substrate, or in the tunnel barriers, or by transients due to the pulsed operation of the qubit.

Alternatively one could create a new type of charge qubit by connecting the Cooper Pair Box island to two JJ tunnel junctions, creating a Single Cooper Pair Transistor (SCT). This could be probed via charge ${ }^{16}$ or current $^{28,29,30,31}$ measurements. These experiments can be designed either as threshold detection measurements or as microwavereflection measurements. The reflection measurements with phase-shift detection will be the main theme of this paper.

Moreover, there is the persistent-current flux qubit, based on a quantum version of the RF-SQUID ${ }^{33.34 .35 .36}$ coupled to a measurement dc-SQUID. This measurement SQUID be operated either as a current threshold detector ${ }^{35.36}$ or as resonance circuit reflecting and phase-shifting microwave radiation ${ }^{20.21}$. We will briefly describe the microwave reflection measurement also in this case in sect. VI.

\section{CHARGE MEASUREMENTS}

The most straightforward way to read out a charge qubit is to measure its charge. As discussed above, to obtain a high fidelity read out one should perform a measurement in the qubit eigenbasis. This removes the possibility for the qubit to switch its state during the measurement. When the measurement basis is fixed, as determined by a charge measurement, we need to bias the qubit where the charge basis is the eigenbasis. For the superconducting charge qubit this implies a complete quenching of the Josephson energy, while for quantum dot charge qubits one needs raise the tunnel barrier between the dots. Having quenched the transitions between the charge states the fidelity will in theory be perfect, irrespectively of the measurement speed. In reality there is always some remaining transition/relaxation channel open which implies the need of a fast read-out. Fast read-out is also mandatory for implementing an error correcting algorithm, where the read out and correction should be performed on a time-scale set by decoherence of the other qubits.

\section{A. The radio-frequency single-electron transistor}

The state of the art charge measurement device is the radio-frequency single-electron transistor (rf-SET) ${ }^{26}$ with an experimentally measured sensitivity of $\delta q=3.2 \cdot 10^{-6} \mathrm{e} / \sqrt{\mathrm{Hz}}{ }^{37}$. The measurement time $t_{m s}$ needed to separate two states with a charge difference $\Delta Q$ is $t_{m s}=(2 \delta q / \Delta Q)^{2}$, indicating the possibility of detecting a charge difference of one percent of the electron charge $(\Delta Q=0.01 \mathrm{e})$ in half a microsecond.

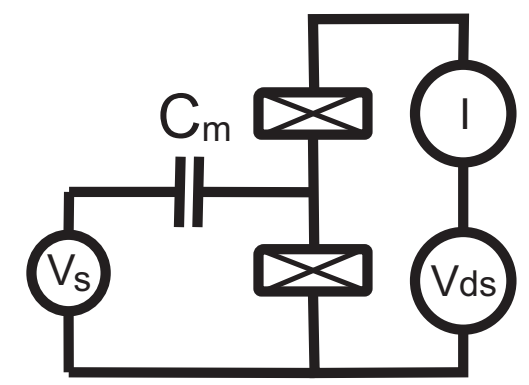

FIG. 2: A single-electron transistor: a small metallic island connected to source and drain leads through tunnel junctions. The signal voltage $V_{s}$ induces a charge $q_{m}$ on the measurement capacitance $C_{m}$. When the system is at the limit of being Coulomb blockaded, a small change of $q_{m}$ will have a large effect on the current $I$ through the SET.

The single-electron transistor (SET) consists of a small metallic island connected to source and drain leads through tunnel junctions (see Fig. 2). Applying a source-drain voltage the current through the SET depends critically on the charge induced on the gate capacitance. The charge on the gate capacitance is determined by measuring the current. The SET is in itself a sensitive electrometer but suffers from low operating speed, which in addition to being a 


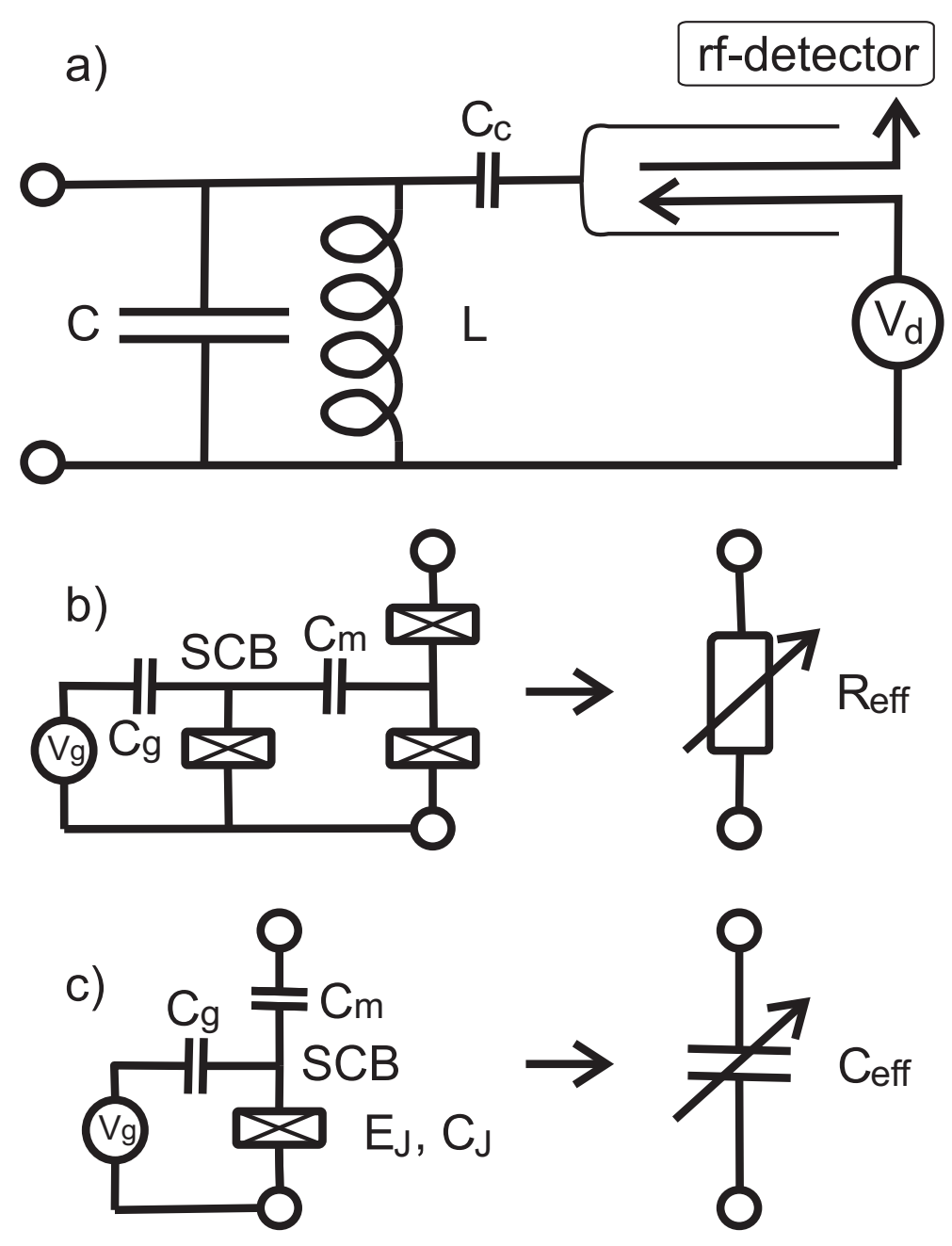

FIG. 3: Resonant circuits for read-out: a) A lumped element LC-oscillator coupled to a driving source and a radio-frequency detector through a transmission line. b) The radio-frequency single-electron transistor measuring the charge of a charge qubit (SCB). The current through the SET determines the dissipation in the resonant circuit. The dissipation is determined by measuring the amplitude of the reflected signal. c) Setup for measuring the quantum capacitance of the charge qubit. The qubit capacitance influences the resonance frequency of the oscillator. The capacitance is measured by determining the phaseshift of the reflected signal.

drawback on its own makes it sensitive to the low frequency charge fluctuations. The rf-SET is realized by embedding an SET in a resonant circuit (see Fig. Fig. 3 a,b). The main source of dissipation in the oscillator is current flowing through the SET, and for small amplitude oscillations we may replace the SET by its effective (differential) resistance. The oscillator is excited by sending down a radio-frequency signal on resonance. The dissipation is determined by measuring the amplitude of the reflected signal. This way of operating the SET increases its operating speed and sensitivity significantly.

\section{B. Single-shot read-out}

The high sensitivity can be used to couple the rf-SET weakly to the charge qubit, reducing the back-action in the off-state. In practice it is impossible to switch off the interaction completely, i.e. the qubit eigenbasis is not exactly the charge basis, and there is some unavoidable mixing of the charge states . A careful investigation of the rf-SET coupled to a superconducting charge qubit shows that single-shot read-out with a very high fidelity is still possible in practice 38.39 .40 . To optimize the fidelity one should bias the charge qubit as far away from the degeneracy point as possible, in order to minimize the effect of any residual Josephson coupling. 


\section{CAPACITANCE MEASUREMENTS - A QUASICLASSICAL DESCRIPTION}

The low-frequency charge fluctuations present in all realizations of superconducting charge qubits so far ${ }^{41.42}$ promotes the use of schemes where the charge qubits are operated at the charge degeneracy point around the origin in Fig. 1 (middle panel). Here the qubit eigenstates have equal average charge and thus they are shielded from charge fluctuations. To use rf-SET read-out described in the previous section one needs to quickly shift the qubit far away from degeneracy simultaneously with switching-on the measurement. Although this timing is far from impossible, the present trend is to use schemes where the qubit remains at charge degeneracy also during read-out in order to minimize decoherence. This can be achieved by measuring the effective capacitance of the charge qubit, as we describe below.

\section{A. Quantum capacitance of a single-Cooper-pair box}

The quantum capacitance of the Cooper-pair box ${ }^{43.44}$ is related to the parametric capacitance of small Josephson junctions 45.46 .47 which is a dual to the Josephson inductance. The origin of the quantum capacitance of a singleCooper-pair box (SCB) can be understood as follows. Assume that we put a constant voltage $V_{m}$ on the measurement capacitance of the SCB, i.e. we put a voltage source between the open circles in Fig. 35. The amount of charge on the measurement capacitance $q_{m}^{g / e}\left(V_{m}, V_{g}\right)$ will be a nonlinear function of the voltage $V_{m}$ as well as the gate voltage $V_{g}$ and whether the qubit is in the ground or excited state. We may define an effective (differential) capacitance

$$
C_{\mathrm{eff}}^{g / e}\left(V_{m}, V_{g}\right)=\frac{\partial}{\partial V_{m}} q_{m}^{g / e}\left(V_{m}, V_{g}\right),
$$

as seen from the measurement circuitry. Away from the charge degeneracy points of the SCB no charge will float across the Josephson junction and the effective capacitance is simply the geometric capacitance $C_{\text {geom }}=C_{J} C_{m} /\left(C_{J}+C_{m}\right)$ of the Josephson junction capacitance $C_{J}$ and the measurement capacitance $C_{m}$ in series. Around the charge degeneracy point a change of voltage will induce a shift of a Cooper-pair across the Josephson junction. For a voltage changing slowly on the timescale of the inverse qubit gap $\hbar E_{J}^{-1}$ this charge redistribution is dissipationless. This contribution to the effective capacitance, which depends on the qubit state, we call the quantum capacitance $C_{Q}^{g / e}$. From the SCB Hamiltonian (see e.g. Eq. (18) ) it is straightforward to show

$$
C_{\mathrm{eff}}^{g / e}\left(V_{m}, V_{g}\right)=\frac{C_{J} C_{m}}{C_{J}+C_{m}} \pm \frac{C_{m}^{2}}{C_{\Sigma}} \frac{E_{J}^{2} E_{Q}}{\left(E_{Q}^{2}\left[1-2\left(n_{g}+n_{m}\right)\right]^{2}+E_{J}^{2}\right)^{3 / 2}}=C_{\mathrm{geom}}+C_{Q}\left(n_{g}+n_{m}\right),
$$

where $C_{\Sigma}=C_{J}+C_{m}+C_{g}$ is the total island capacitance, $E_{Q}=2 e^{2} / C_{\Sigma}$ is the Coulomb energy of a Cooper-pair, and $n_{g / m}=C_{g / m} V_{g / m} / 2 e$ are the induced number of Cooper-pairs on the gate and measurements capacitances respectively. We note that the quantum capacitance is positive in the ground state and negative in the excited state, as illustrated in Fig. 4. The absolute value is largest at the charge degeneracy $n_{g}+n_{m}=0.5$

$$
C_{Q}^{\max }=\frac{C_{m}^{2}}{C_{\Sigma}} \frac{E_{Q}}{E_{J}}
$$

The quantum capacitance can be an order of magnitude larger than the geometric capacitance for realistic parameters. By inserting the Cooper-pair box in a resonant LC-circuit and detect its influence on the resonance frequency, the quantum capacitance was recently measured by two different groups ${ }^{15.16}$. The measurement setup is similar to the setup that would be used for qubit read-out and is analyzed below.

\section{B. Read-out by measuring the quantum capacitance}

At the charge degeneracy point the effective capacitance of the SCB in the ground and excited state differs by $2 C_{Q}^{\max }$. Imbedding the $\mathrm{SCB}$ in a resonant circuit as shown in Fig. [3 a) and c) we can detect the corresponding change in the oscillators resonance frequency $\omega_{0}^{g / e}=1 / \sqrt{L\left(C \pm C_{Q}^{\max }\right)}=\omega_{0}\left(1 \mp C_{Q}^{\max } / 2 C\right)$, where $\omega_{0}=1 / \sqrt{L C}$ is the bare resonance frequency. The voltage reflection amplitude $\Gamma(\omega)=V_{\text {out }}(\omega) / V_{d}(\omega)$ seen from the driving side of 


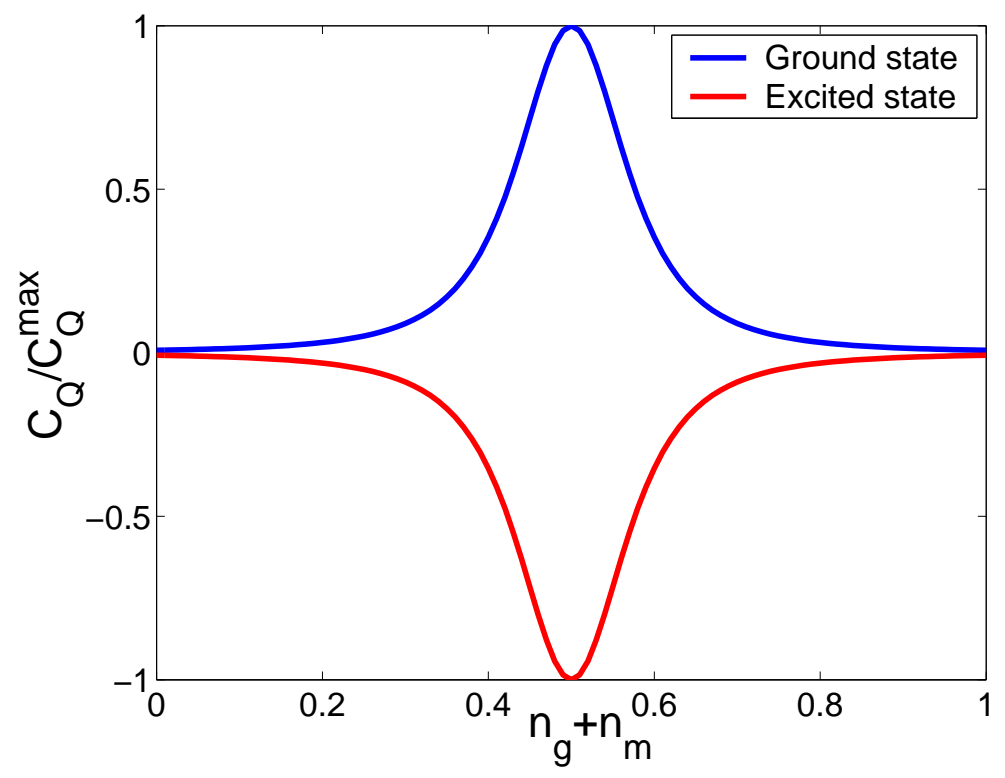

FIG. 4: The quantum capacitance of a single-Cooper-pair box with $E_{J} / E_{Q}=0.2$. Here normalized by the maximum value $C_{Q}^{\max }=\frac{C_{m}^{2}}{C_{\Sigma}} \frac{E_{Q}}{E_{J}}$

the transmission line can for a high quality oscillator be written

$$
\Gamma(\omega)=\frac{1+2 i Q \frac{\left(\omega-\omega_{0}\right)}{\omega_{0}}}{1-2 i Q \frac{\left(\omega-\omega_{0}\right)}{\omega_{0}}}=e^{i \varphi_{r}}, \text { where } \varphi_{r}=2 \arctan \left(\frac{2 Q\left(\omega-\omega_{0}\right)}{\omega_{0}}\right),
$$

up to a constant phase depending on the length of the transmission line. Here $Q$ is the resonator's quality factor, which for the circuitry in Fig. 3] a) is determined by the characteristic impedance on the transmission line $Z_{0}$ through $Q=\omega_{0} L C^{2} / C_{c}^{2} Z_{0}$. Since there is no dissipation in the oscillator we have $|\Gamma(\omega)|=1$. Driving the oscillator at the bare resonance frequency $\omega_{d}=\omega_{0}$ the phase-difference between the ground and excited state of the qubit will be

$$
\delta \varphi_{r}=\varphi_{r}^{g}-\varphi_{r}^{e}=4 \arctan \left(Q C_{Q}^{\max } / C\right)
$$

The phase-difference can be detected by measuring the reflected signals in-phase and quadrature components after mixing it with the drive.

\section{A QUANTUM DESCRIPTION OF THE QUANTUM CAPACITANCE READOUT}

Above we described the quantum capacitance of the Cooper-pair box and its use for qubit read-out in a quasiclassical manner, treating the oscillator, transmission line, drive and detection classically. In order to address questions about the optimal read-out time, quantum back-action on the qubit and the quantum efficiency of the read-out process we need a fully quantum description of the system. In this paper we do not have the space to go into details, which will be published elsewhere ${ }^{48}$, but we will discuss the principles of our model and show a fully quantum derivation of the quantum capacitance.

The approach we chose is close to the "Quantum Network Theory" introduced by Yurke and Denker ${ }^{49}$. In section $\nabla A$ we start by writing down the Lagrangian describing the classical dynamics of the circuit. Through a Legendre transform we arrive at the corresponding Hamiltonian. By stating canonical commutation relations between our phase coordinates $\Phi_{i}$ and their canonical conjugate momenta (charges) $q_{i}$

$$
\left[\Phi_{i}, q_{i}\right]=i \hbar
$$

we arrive at a quantum Hamiltonian description of our circuit, which is discussed in section $\nabla \mathrm{B}$ In the relevant parameter regime we arrive at the expression for the quantum capacitance of the Cooper-pair box. Finally in section $\mathrm{VC}$ we give an expression for the optimal qubit read-out time using homodyne detection. 


\section{A. Circuit Lagrangian}

The circuit for performing read-out through the quantum capacitance is presented in figure 5] A Josephson charge qubit is capacitatively coupled to a harmonic oscillator, which is coupled to a transmission line. Through this line, all measurement on the qubit is performed. We model the line as a semi-infinite line of LC-circuits in series. The working point of the Josephson junction can be chosen using the bias $V_{g}$. In writing down the Lagrangian we are free to chose any quantities as our coordinates as long as they give a full description of our circuit. Since we are treating a system including a Josephson junction, the phases $\Phi_{i}(t)=\int^{t} d t^{\prime} V_{i}\left(t^{\prime}\right)$ across the circuit elements are natural coordinates, as discussed by Devoret in ref. 50. (This is in contrast to the original work by Yurke and Denker where charges are chosen as coordinates.)

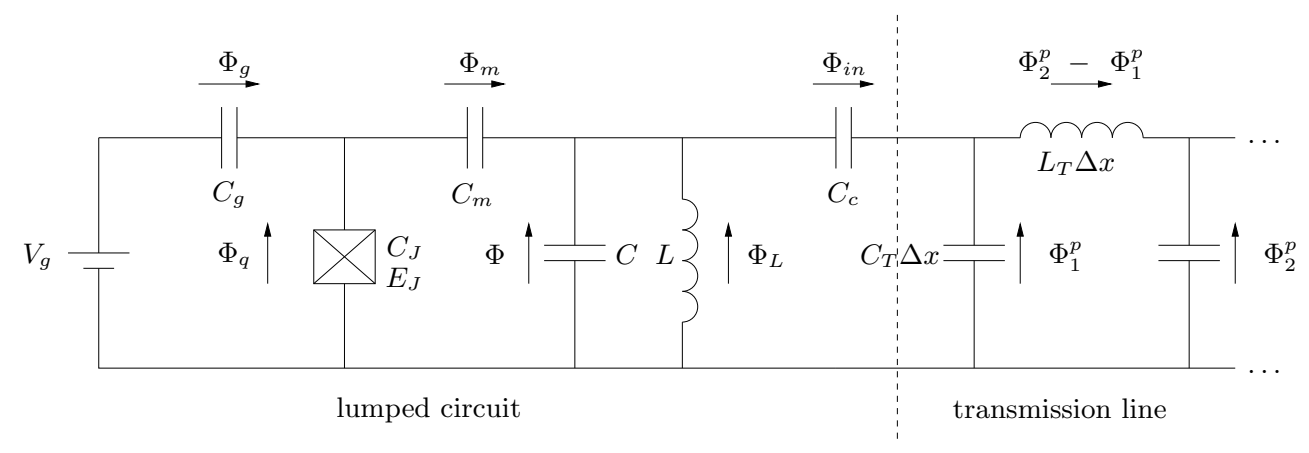

FIG. 5: The circuit used for measurement of the quantum capacitance of the Cooper-pair box. It is similar to the circuit shown in Fig. $3 \mathrm{~B})+\mathrm{c}$ ), but the transmission line is here modelled as a semi-infinite line of LC-circuits in series. The phases $\Phi_{i}(t)=\int^{t} d t^{\prime} V_{i}\left(t^{\prime}\right)$ across the different circuit elements are the coordinates used in the Lagrangian describing the dynamics of the system.

The capacitive energy of the circuit act as the kinetic terms in the Lagrangian 3

$$
T=\frac{1}{2}\left(C_{g} \dot{\Phi}_{g}^{2}+C_{J} \dot{\Phi}_{J}^{2}+C_{m} \dot{\Phi}_{m}^{2}+C \dot{\Phi}^{2}+C_{c} \dot{\Phi}_{i n}^{2}\right)+\frac{1}{2} \sum_{i=1}^{\infty} \Delta x C_{T}\left(\dot{\Phi}_{i}^{p}\right)^{2}
$$

and the inductive part plays the part of potential energy

$$
V=\frac{\Phi_{L}^{2}}{2 L}-E_{J} \cos \left(\frac{2 e}{\hbar} \Phi_{J}\right)+\sum_{i} \Delta x \frac{\left(\Phi_{i+1}^{p}-\Phi_{i}^{p}\right)^{2}}{2 L_{T}(\Delta x)^{2}}
$$

Applying Kirchoff's voltage law gives us the constraints

$$
\begin{array}{r}
\dot{\Phi}_{g}-\dot{\Phi}_{J}+V_{g}=0, \Phi_{J}+\Phi_{m}-\Phi=0 \\
\Phi_{i n}+\Phi-\Phi_{1}^{p}=0, \Phi-\Phi_{L}=0
\end{array}
$$

which gives the Lagrangian for the system

$$
\begin{aligned}
L & =\frac{C_{q b} \dot{\Phi}_{J}^{2}}{2}+\frac{\left(C_{o s c}+C_{c}\right) \dot{\Phi}^{2}}{2}+\frac{C_{c}\left(\dot{\Phi}_{1}^{p}\right)^{2}}{2}-\frac{\Phi^{2}}{2 L}+E_{J} \cos \left(\frac{2 e}{\hbar} \Phi_{J}\right)-\frac{C_{m}}{2} \dot{\Phi}_{J}-\frac{C_{c}}{2} \dot{\Phi}_{1}^{p}-C_{g} V_{g} \dot{\Phi}_{J}+ \\
& +\sum_{i=1}^{\infty} \Delta x\left(\frac{C_{T}\left(\dot{\Phi}_{i}^{p}\right)^{2}}{2}-\frac{\left(\Phi_{i+1}^{p}-\Phi_{i}^{p}\right)^{2}}{2 L_{T}(\Delta x)^{2}}\right)
\end{aligned}
$$

The capacitances in the Lagrangian are now $C_{q b}=C_{J}+C_{g}+C_{m}$, and $C_{o s c}=C+C_{m}$.

\section{B. Hamiltonian and quantum capacitance}

From the Lagrangian we easily obtain the Hamiltonian through a Legendre transform. We present the Hamiltonian on the form

$$
H=H_{q b}+H_{o s c}+H_{T L}+H_{i n t}
$$


and for simplicity we assume weak coupling $C_{m} \ll\left\{C_{o s c}, C_{q b}\right\}$ and present only the lowest order (in $C_{m} /\left\{C_{q b}, C_{o s c}\right\}$ ) terms. $H_{q b}$ contains the qubit degrees of freedom including the coupling of the qubit to the rest of the system

$$
H_{q b}=\frac{1}{2 C_{q b}}\left(q_{J}+C_{g} V_{g}\right)^{2}+\frac{C_{m}}{C_{q b} C_{o s c}}\left(q+q_{p}\right)\left(q_{J}+C_{g} V_{g}\right)-E_{J} \cos \left(\frac{2 e}{\hbar} \Phi_{J}\right),
$$

while the terms describing the oscillator, transmission line and their interaction are

$$
H_{o s c}=\frac{q^{2}}{2 C_{o s c}}+\frac{\Phi^{2}}{2 L}, \quad H_{T L}=\frac{q_{p}^{2}}{2 C_{c}}+\frac{1}{\Delta x} \sum_{i=1}^{\infty}\left(\frac{\left(q_{(i+1)}^{p}\right)^{2}}{2 C_{T}}+\frac{\left(\Phi_{i+1}^{p}-\Phi_{i}^{p}\right)^{2}}{2 L_{T}}\right), \quad H_{\text {int }}=\frac{q q_{p}}{C_{o s c}},
$$

where the charge operators $q, q_{J}, q_{p}$ and $q_{i}^{p}$ are the conjugate momenta to phase operators $\Phi, \Phi_{J}, \Phi_{1}^{p}$ and $\Phi_{i}^{p}$ respectively. For realizing a charge qubit the box charging energy $E_{C}=e^{2} / 2 C_{q b}$ is much smaller than the Josephson energy $E_{C} \gg E_{J}$. For relevant parameters we can then limit the qubit charge $q_{J}$ to the two values $\{0,2\}$ e, and we get the usual expression for the qubit Hamiltonian in the language of the Pauli spin matrices

$$
H_{q b}=-\frac{E_{e l}}{2} \sigma_{z}-\frac{E_{J}}{2} \sigma_{x}+2 E_{C} \kappa \frac{q+q_{p}}{e} \sigma_{z}+2 E_{C} \kappa \frac{q+q_{p}}{e}\left(1-n_{0}\right),
$$

where we introduce the electrostatic energy-difference of the qubit states $E_{e l}=4 E_{C}\left(1-n_{0}\right)$, the dimensionless charge $n_{0}=C_{g} V_{g} / e$, and the oscillator-qubit coupling coefficient $\kappa=C_{m} / C_{o s c} \ll 1$. The last term does not influence the systems dynamics and may be absorbed in a small shift of $q$ and $q_{p}$. Rotating the remaining two terms to the eigenbasis of the qubit

$$
H_{q b}=\sigma_{z} \sqrt{16 E_{C}^{2}\left(1-n_{0}-\kappa\left(q+q_{p}\right) / e\right)^{2}+E_{J}^{2} / 4}
$$

we arrive at the usual charge qubit Hamiltonian with the charge induced by the oscillator added to the induced gate charge. We now concentrate on the case when the oscillator frequency $\omega_{0}=1 / \sqrt{L C_{o s c}}$ is much lower than qubit frequency $E_{J} / \hbar$. Furthermore we consider the amplitude of the oscillators charge oscillations $q_{0}$ such that the induced charge oscillations in the qubit are small $\kappa q_{0} / e \ll E_{J} / 4 E_{C} \ll 1$. In this case the qubit will follow the oscillator dynamics adiabatically and the rates for transition between the qubit eigenstates are negligible. Furthermore, we may Taylor expand the qubit energy around the working point and at the charge degeneracy $n_{0}=1$ we arrive at the final Hamiltonian

$$
H=-\frac{E_{J}}{2} \sigma_{z}+\left(\frac{1}{2 C_{o s c}}+\frac{4 \kappa^{2} E_{C}^{2}}{e^{2} E_{J}} \sigma_{z}\right)\left(q+q_{p}\right)^{2}+\frac{\Phi^{2}}{2 L}+\frac{q_{p}^{2}}{2 C_{c}}+\frac{1}{\Delta x} \sum_{i=1}^{\infty}\left(\frac{\left(q_{i+1}^{p}\right)^{2}}{2 C_{T}}+\frac{\left(\Phi_{i+1}^{p}-\Phi_{i}^{p}\right)^{2}}{2 L_{T}}\right) .
$$

The qubit thus shifts the capacitative energy of the oscillator, which in turn corresponds to adding a small extra capacitor to the oscillator $C_{\Sigma}=C_{o s c}+C_{Q}$, where the quantum capacitance $C_{Q}$ is given by

$$
C_{Q}=-\frac{2 e^{2} C_{m}^{2}}{E_{J} C_{q b}^{2}} \sigma_{z}
$$

which is identical to the semiclassical formula in Eq. [6] This in turn will shift the resonance frequency of the oscillator with an amount $\delta \omega=-\sigma_{z} \omega_{0} C_{Q} / 2 C_{o s c}$.

\section{Qubit read-out using homodyne detection}

Taking the continuum limit $\Delta x \rightarrow 0$ in Eq. (19) the solutions to the Hamiltonian for the transmission line correspond to fields $\Phi(x \pm v t)$ propagating to the left and right with velocity $v=1 / \sqrt{C_{T} L_{T}}$. From the Hamiltonian we derive equations of motion from which the right-propagating out-field $\Phi^{\text {out }}(t)$ and the charge on the oscillator $q(t)$ can expressed in terms of the left-propagating in-fields $\Phi^{i n}(t)$, which is determined by the drive. In the parameter regime relevant for qubit read-out, where the amplitude of the qubit charge oscillations induced by the LC-circuit is small, the qubit will follow the oscillator adiabatically. Furthermore it is adequate to neglect third and higher order terms in the Taylor expansion of the qubit energy in Eq. (18). Thus we arrive at linear equations of motion which we may solve in Fourier representation

$$
\begin{aligned}
\chi(\omega) & =\frac{i 2 C_{c} C_{\Sigma} L \omega^{3}}{1-\left(C_{\Sigma}+C_{c}\right) L \omega^{2}-i \omega C_{c} Z_{0}\left(1-L C_{\Sigma} \omega^{2}\right)}, \\
q(\omega)+q_{p}(\omega) & =\chi(\omega) \Phi_{p}^{i n}(\omega), \\
\Phi^{\text {out }}(\omega) & =\frac{\chi(\omega)}{\chi^{*}(\omega)} \Phi_{p}^{i n}(\omega)=S(\omega) \Phi_{p}^{i n}(\omega),
\end{aligned}
$$


where $Z_{0}=\sqrt{L_{T} / C_{T}}$ is the characteristic impedance of the transmission line. Since there is no dissipation in the lumped circuit we have $\left|\Phi^{\text {out }}(\omega)\right|=\left|\Phi^{i n}(\omega)\right|$. In this linear regime the Heisenberg equations of motion are similar to the classical ones and for a high quality oscillator we have $S(\omega)=\Gamma(\omega)$ as given in Eq. (7). To discuss the quantum statistics of the qubit read-out we need a quantized representation of the fields

$$
\begin{aligned}
\Phi^{i n}(t) & =\sqrt{\frac{\hbar Z_{0}}{4 \pi}} \int_{0}^{\infty} \frac{d \omega}{\sqrt{\omega}}\left[a_{\omega}^{i n} e^{-i \omega t}+\left(a_{\omega}^{i n}\right)^{\dagger} e^{i \omega t}\right], \\
\Phi^{\text {out }}(t) & =\sqrt{\frac{\hbar Z_{0}}{4 \pi}} \int_{0}^{\infty} \frac{d \omega}{\sqrt{\omega}}\left[S(\omega) a_{\omega}^{i n} e^{-i \omega t}+S(\omega)^{*}\left(a_{\omega}^{i n}\right)^{\dagger} e^{i \omega t}\right], \\
q(t) & =\sqrt{\frac{\hbar Z_{0}}{4 \pi}} \int_{0}^{\infty} \frac{d \omega}{\sqrt{\omega}}\left[\chi(\omega) a_{\omega}^{i n} e^{-i \omega t}+\chi(\omega)^{*}\left(a_{\omega}^{i n}\right)^{\dagger} e^{i \omega t}\right],
\end{aligned}
$$

where the in-field annihilation and creation operators obey the canonical commutation relations

$$
\left[a_{\omega}, a_{\omega^{\prime}}^{\dagger}\right]=\delta\left(\omega-\omega^{\prime}\right) \text {, and }\left[a_{\omega}, a_{\omega^{\prime}}\right]=0 .
$$

What we have achieved is a complete quantum description of the dynamics of the oscillator and qubit in terms of the incident field from the transmission line. We also get a full description of the outgoing field, which is what will enter the detector. The linearity of the equations of motion arise since we have approximated the qubit with a state-dependent capacitance. This is valid as long as the amplitude of the charge oscillations induced on the qubit island are small, so that a second order Taylor expansion of the energy in Eq. (18)is enough. Furthermore we neglect transitions between the qubit states, which can be done for small amplitude oscillations and a low oscillator frequency $\hbar \omega_{0} \ll E_{J}$.

We have now the formalism needed to address questions about the readout time which we do in the next section. We can also calculate the backaction of the measurement process on the qubit. This and the issue of the quantum efficiency, i. e. the relation between the qubit dephasing rate and the measurement time will be addressed elsewhere $\underline{\underline{48}}$.

By measuring the power of the reflected signal mixed with a local oscillator, e.g. the drive itself, and then a local oscillator shifted 90 degrees the in-phase and quadrature signal amplitude can be extracted, as was done in Ref. 25. For optimized qubit read-out it's advantageous to implement the standard quantum optics scheme of homodyne detection ${ }^{52}$.

To model a measurement we thus put the in-field in a coherent Glauber state ${ }^{51}$

$$
|\{\alpha(\omega)\}\rangle=\exp \left(\int d \omega\left[\alpha(\omega)\left(a_{\omega}^{i n}\right)^{\dagger}-\alpha^{*}(\omega) a_{\omega}^{i n}\right]\right)|0\rangle,
$$

where $\alpha(\omega)$ is the Fourier-transform of our drive signal, and $|0\rangle$ is the continuum vacuum field $a_{\omega}|0\rangle=0$. We model our drive source with a narrow $\left(\Gamma_{d} \ll \omega_{d}\right)$ Gaussian distribution in frequency

$$
\alpha(\omega)=\alpha_{0} \frac{\omega_{d}}{\Gamma_{d}} \frac{e^{-\left(\omega-\omega_{d}\right)^{2} / 2 \Gamma_{d}^{2}}}{\sqrt{\omega}},
$$

where $\alpha_{0}$ is a dimensionless constant. For $|t| \ll \Gamma_{d}^{-1}$ this gives the average electrical field

$$
\bar{V}^{i n}(x, t)=-\alpha_{0} \sqrt{2 \hbar Z_{0}} \omega_{d} \sin \left[\omega_{d}(t+x / v)\right],
$$

giving on average $\Gamma_{n}^{i n}=\alpha_{0}^{2} \omega_{d}$ photons per second sent through the transmission line by the drive.

The annihilation operator for the reflected signal has the amplitude $a_{\omega}^{\text {out }}=S(\omega) a_{\omega}^{i n} \approx e^{-\left(\omega_{d} t-\varphi_{r}^{g / e}\right)} a_{\omega}^{i n}$ depending on the qubit state. The signal is mixed with a strong local oscillator with amplitude $\alpha_{L O} e^{-\left(\omega_{d} t-\varphi_{L O}\right)}$ and the intensity is detected. The result is then integrated for a time $T$. The intensity is given by the number of photons incident on the detector

$$
N(\omega, T)=\int_{0}^{T} d t b^{\dagger}(t) b(t)
$$

where the field at the detector is

$$
b(t)=r \alpha_{L O}(t)+r v(t)+t a^{o u t}(t),
$$


here $r \ll 1$ denotes the small reflection coefficient of the mixer and $t$ is the corresponding transmission coefficient, and $v(t)$ is the vaccuum-field of the idle mixer port. (The mixer is a beam-splitter in the quantum optics case.) We now assume that $\left|r \alpha_{L O}(t)\right| \gg 1$ is large and neglect second order contributions in $v(t)$ and $a(t)$ to equation (27), arriving at the average photon number at the detector

$$
\left\langle N^{g / e}\right\rangle=T\left(r^{2} \alpha_{L O}^{2}+2 \operatorname{tr} \alpha_{L O} \alpha_{0}^{i n} \cos \left(\varphi_{r}^{g / e}-\varphi_{L O}\right)\right)
$$

where $\alpha_{0}^{i n}$ is amplitude of the in-field. The first term is a pure local oscillator term and contains no information, while the second term is maximized choosing $\varphi_{L O}=\frac{\varphi_{r}^{g}+\varphi_{r}^{e}}{2}-\frac{\pi}{2}$. The two probability distributions of the number of detected photons will be separated by two times the variance after the measurement time

$$
T_{m s}=\frac{1}{\Gamma_{n}^{i n}} \frac{1}{4 \sin ^{2}\left[\frac{\varphi_{r}^{g}-\varphi_{r}^{e}}{2}\right]},
$$

where $\Gamma_{n}^{i n}$ is rate of photons sent into the transmission line by the drive. For not too low quality factor of the oscillator we can use the Breit-Wigner approximation for $\chi(\omega)$ leading to the expression for the phase-shift in Eq. (8). Thus we find for the measurement time

$$
T_{m s}=\frac{1}{\Gamma_{n}^{i n}} \frac{1}{4 \sin ^{2}[2 \arctan x]}=\frac{\left(x^{-1}+x\right)^{2}}{16 \Gamma_{n}^{i n}}, x=\frac{Q C_{Q}^{\max }}{C} .
$$

From the condition that the drive should induce only small oscillations of the qubit charge we arrive at the following bound for the drive strength

$$
\Gamma_{n}^{i n}<\frac{E_{J}}{16 \hbar}\left(x+x^{-1}\right)
$$

giving a lower bound on measurement time

$$
T_{m s}>\frac{\hbar}{E_{J}}\left(x+x^{-1}\right)
$$

indicating that the measurement time must be larger than $\hbar / E_{J}$, which is not very restrictive. Due to the oscillator "ring-up" time the measurement time is further limited by $T_{m s}>Q / \omega_{0}=x C / C_{Q} \omega_{0}$. For a fixed $\omega_{0}$ this indicates that the regime $x \ll 1$ is advantageous. Comparing these two inequalities we find the shortest measurement time for

$$
x_{o p t}^{2}=\frac{C_{Q}}{C} \frac{\hbar \omega_{0}}{E_{J}}, \quad Q_{o p t}=\sqrt{\frac{C}{C_{Q}} \frac{\hbar \omega_{0}}{E_{J}}}, \text { and } T_{m s}>\frac{Q_{o p t}}{\omega_{0}},
$$

implying that a low $Q$ is clearly an interesting regime. For low $Q$ the Breit-Wigner approximation of $\chi(\omega)$ breaks down, and so does the simple estimates of the measurement, but the formalism developed here is still applicable using the full expressions. An optimization including the measurement induced back-action on the qubit will be discussed in Ref. 48 .

\section{A comparison with dispersive readout using a non-adiabatic oscillator}

In an experiment at Yale university ${ }^{53}$ a charge qubit coupled capacitatively to a microstrip cavity was read out by sending microwaves through the cavity. The qubit state influences the resonance frequency and thus the phase-shift of the transmitted signal. This phase difference was then detected in a similar fashion as described above.

The main difference compared to what was discussed above is that the cavity resonance frequency $(5.4 \mathrm{GHz})$ was of the same order of magnitude as the qubit frequency $(4.3 \mathrm{GHz})$. In this regime it is appropriate to use the rotating wave approximation and the system dynamics is described by the Jaynes-Cummings hamiltonian 10 .

For a comparison we start with the Hamiltonian of a qubit coupled transversely to a harmonic oscillator

$$
H=-\frac{\hbar \omega_{q b}}{2} \sigma_{z}+i g \sigma_{x}\left(a^{\dagger}-a\right)+\hbar \omega_{o s c}\left(a^{\dagger} a+\frac{1}{2}\right),
$$


applicable for a charge qubit at charge degeneracy, coupled capacitatively to the harmonic oscillator. Performing a straightforward second order perturbation expansion in the coupling term we find the renormalized spectrum

$$
\begin{aligned}
& E_{n \uparrow}=\hbar \omega_{o s c}\left(n+\frac{1}{2}\right)-\frac{\hbar \omega_{q b}}{2}-g^{2}\left[\frac{n}{\hbar \omega_{q b}-\hbar \omega_{o s c}}+\frac{n+1}{\hbar \omega_{q b}+\hbar \omega_{o s c}}\right] \text { and } \\
& E_{n \downarrow}=\hbar \omega_{o s c}\left(n+\frac{1}{2}\right)+\frac{\hbar \omega_{q b}}{2}+g^{2}\left[\frac{n}{\hbar \omega_{q b}+\hbar \omega_{o s c}}+\frac{n+1}{\hbar \omega_{q b}-\hbar \omega_{o s c}}\right],
\end{aligned}
$$

where $n$ indicates the number of photons in the oscillator and $\uparrow / \downarrow$ the qubit in the ground/excited state for the unperturbed state $(g \rightarrow 0)$. The spectrum is formed by two equidistant sets of energy levels, where the effective qubit-dependent frequency shift of the oscillator amounts to

$$
\delta \omega=-\sigma_{z} g^{2}\left[\frac{1}{\hbar \omega_{q b}-\hbar \omega_{o s c}}+\frac{1}{\hbar \omega_{q b}+\hbar \omega_{o s c}}\right],
$$

being negative for the qubit in the ground state. In the regime $\left|\omega_{q b}-\omega_{o s c}\right| \ll \omega_{q b}$ only the first term contributes and we arrive at the Jaynes-Cummings result $\delta \omega=-\sigma_{z} g^{2} /\left(\hbar \omega_{q b}-\hbar \omega_{o s c}\right)$. In the adiabatic regime $\omega_{o s c} \ll \omega_{q b}$ we can neglect the terms $\pm \hbar \omega_{\text {osc }}$ in the denominators giving the result below Eq. (20), $\delta \omega=-\sigma_{z} 2 g^{2} / \hbar \omega_{q b}$. Thus we see that the frequency shift given by the Jaynes-Cummings hamiltonian can be described on the same footing as the one given by the quantum capacitance.

\section{FLUX MEASUREMENT}

The 2-level quantum states of the persistent-current flux qubit (Fig. 1) are characterized by different directions of the persistent currents circulating in the qubit loop, hence different directions of the induced magnetic flux. The flux qubit readout is based on the detection of the induced flux or direct measurement of the persistent currents. The latter method is also relevant for charge qubits with loop-shape electrodes (e.g. quantronium ${ }^{28}$ ) where the intensity of the induced flux is too small to be detectable while detection of the persistent current is possible. In flux qubits with larger Josephson junctions, persistent currents are large, and the measurement of flux is not that difficult 18.20 .35 .55 . The experimental measurement setup is sketched in Fig. 1, left picture: the qubit loop is inductively coupled to a dc-SQUID connected to a current source. Direction of the persistent current in the qubit loop affects the magnetic flux threading the SQUID and thus affects the SQUID critical current as well as its plasma frequency. This allows one to make the two types of measurements, by probing the dc and the ac properties of the measurement SQUID. In the first case, the critical current is measured by applying a dc current slowly increasing with time, and detecting the value of the critical current when the SQUID switches to the resistive branch (threshold detection), repeating the measurement to create a histogram of the events. In the second case, an ac current is applied and the phase shift of the reflected signal is measured. The latter method is also possible to realize using a linear LC-oscillator instead of the dc SQUID ${ }^{18}$.

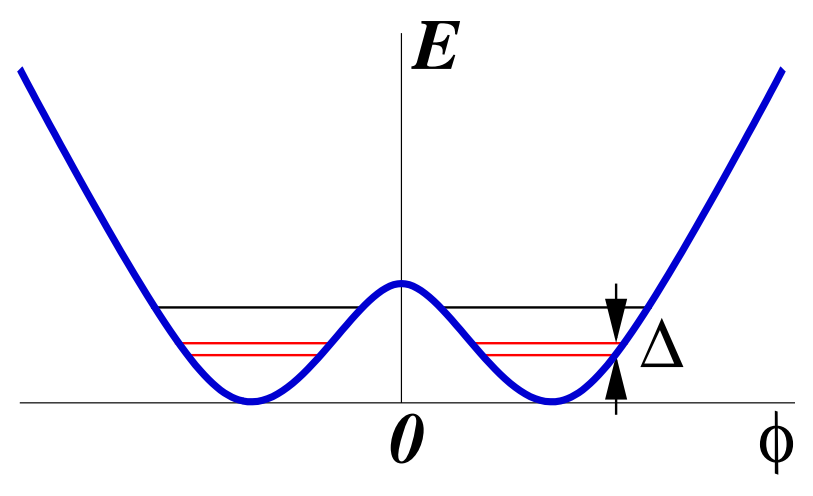

FIG. 6: Double-well potential and energy levels of the flux qubit $\left(f_{q}=\pi\right)$.

To quantitatively analyze the circuit (see e.g. Refs. 3. 21 54), let us for simplicity consider the single junction flux qubit, the analysis also applies to the experimental three junction qubits. The circuit Hamiltonian consists of the 
Hamiltonian $H_{q}$ of the qubit loop, the Hamiltonian of the SQUID, $H_{S}$, and the Hamiltonian of the inductive coupling, $H_{\text {int }}$. The qubit Hamiltonian has the form given in Eq. (2), here we modify the notations,

$$
H_{q}=E_{C q} n_{q}^{2}-E_{J q} \cos \phi_{q}+E_{L q} \tilde{\phi}_{q}^{2}
$$

where $E_{C q}=(2 e)^{2} / 2 C_{q}$ is the charging energy of the qubit junction, $E_{J q}$ is the Josephson energy, $E_{L q}=$ $\left(\Phi_{0} / 2 \pi\right)^{2}\left(1 / 2 L_{q}\right)$ is the inductive energy of the qubit loop, and $\tilde{\phi}_{q}=\phi_{q}-f_{q}$ is the induced flux, $f_{q}$ is the biasing flux. The SQUID Hamiltonian is given by equation,

$$
H_{s}=\sum_{i}^{2}\left(E_{C s} n_{i}^{2}-E_{J s} \cos \phi_{i}\right)+E_{L s} \phi_{s}^{2}+\frac{\hbar}{4 e} I(t)\left(\phi_{1}-\phi_{2}\right),
$$

where the induced flux is $\phi_{s}=\phi_{1}+\phi_{2}-f_{s}$, and $I(t)$ is a (non)stationary current bias. The interaction term has the form

$$
H_{\text {int }}=E_{M} \tilde{\phi}_{q} \phi_{s}
$$

where the interaction energy is determined by the mutual inductance $M, E_{M}=\left(\Phi_{0} / 2 \pi\right)^{2}\left(M / L_{q} L_{s}\right)$.

Now we truncate the Hilbert space of the circuit Hamiltonian to the lowest energy states, which include the two almost degenerate (for $E_{C q} \ll E_{J q}$ and at $f_{q} \approx \pi$ ) lowest energy states in the potential wells of the qubit potential energy, Fig. 6, and the ground state of the SQUID. Then the qubit Hamiltonian takes the form, in the eigenbasis of the non-coupled wells

$$
H_{q}=-\frac{1}{2}\left(\epsilon \sigma_{z}+\Delta \sigma_{x}\right)
$$

Here $\epsilon\left(f_{q}\right)$ is the energy level difference in the wells proportional to $f_{q}-\pi$, and $\Delta$ is the energy level splitting due to the macroscopic tunneling between the wells. The truncated interaction term takes the form,

$$
H_{\text {int }}=E_{M} \phi_{0} \phi_{s} \sigma_{z}
$$

where $\phi_{0}$ is the half distance between the minima of the potential energy. The off-diagonal term in the interaction is neglected since it is exponentially small.

To truncate the SQUID Hamiltonian, we introduce new variables, $\phi_{ \pm}=(1 / 2)\left(\phi_{1} \pm \phi_{2}\right)$, and $\phi_{s}=2 \phi_{+}-f_{s}$,

$$
H_{s}=\frac{1}{2} E_{C s} n_{-}^{2}+2 E_{C s} n_{s}^{2}-2 E_{J s} \cos \phi_{-} \cos \left(\frac{f_{s}+\phi_{s}}{2}\right)+E_{L s} \phi_{s}^{2}+\frac{\hbar}{2 e} I(t) \phi_{-} .
$$

Now we assume that the inductive energy is sufficiently large, $E_{L s} \gg E_{J s}$, to provide small fluctuation of the induced flux, $\phi_{s} \ll 1$. This allows us to expand the cosine term; then keeping the first order term with respect to $\phi_{s}$ (nonvanishing for $f_{s} \neq 0$ ), and taking into account the interaction term 42 we write the $\phi_{s}$-dependent part of the total Hamiltonian on the form,

$$
H\left(\phi_{s}\right)=2 E_{C s} n_{s}^{2}+\left(E_{J s} \cos \phi_{-} \sin \left(\frac{f_{s}}{2}\right)+E_{M} \phi_{0}\left(f_{q}\right) \sigma_{z}\right) \phi_{s}+E_{L s} \phi_{s}^{2} .
$$

This is the linear oscillator shifted from the origin, the shift being proportional to the induced flux in the qubit loop. Making projection on the ground state of this Hamiltonian, we arrive at the non-trivial, qubit-dependent part having the form,

$$
H\left(\phi_{s}\right) \rightarrow-2 E_{J s} \lambda \sin \left(\frac{f_{s}}{2}\right) \sigma_{z} \cos \phi_{-}, \quad \lambda=\frac{E_{M} \phi_{0}}{8 E_{L s}} .
$$

Combining this with the rest of the SQUID Hamiltonian and the truncated qubit Hamiltonian, we finally get:

$$
H=-\frac{1}{2}\left(\epsilon \sigma_{z}+\Delta \sigma_{x}\right)+\frac{1}{2} E_{C s} n_{-}^{2}-2 E_{J s}\left[\cos \left(\frac{f_{s}}{2}\right)+\lambda \sin \left(\frac{f_{s}}{2}\right) \sigma_{z}\right] \cos \phi_{-}+\frac{\hbar}{2 e} I(t) \phi_{-} .
$$

For the 3 -junction qubit ${ }^{32}$ the coupling constant $\lambda$ in Eq. (45) acquires an additional factor ${ }^{56} E_{J q} / E_{L q}$, which results from tracing out the plasma mode in the qubit loop. This mode does not form the qubit in the 3 -junction circuit (in 
contrast to the single-junction qubit), but this mode is an auxiliary one connecting the qubit to the outside world, and it is eliminated similar to the SQUID variable $\phi_{+}$in Eqs. (44) and (45).

The Hamiltonian (46) describes a flux qubit directly coupled via an effective coupling constant $\lambda$ to a non-linear Josephson oscillator. The coupling affects the Josephson energy of the oscillator; hence the critical bias current, i.e. the the magnitude of the bias dc current at which the oscillator switches to the dissipative regime. Quantitatively, these critical current values for the 3-junction qubit are,

$$
\frac{\hbar}{2 e} I_{c}=2 E_{J s}\left(\cos \left(f_{s} / 2\right) \pm \lambda \frac{E_{J q}}{E_{L q}} \sin \left(f_{s} / 2\right)\right)
$$

The advantage of this method is that the measurement circuit can be disconnected during the time period between the measurements by switching off the flux through the SQUID, $f_{s}=0$, thus enhancing the decoherence time of the qubit. The disadvantage of the method is that for slow readout (low Josephson plasma frequency of the SQUID compared to the qubit frequency) the switching current depends on the average value of the induced flux, $\left\langle\sigma_{z}\right\rangle$, which equals zero at the degeneracy point, $\epsilon=0$. Thus measurement can only be performed by departing from the degeneracy point, which is undesirable due to enhanced decoherence. This difficulty can be solved by probing the qubit quantum inductance, which is analogous to the quantum capacitance measurement for charge qubits.

\section{INDUCTANCE MEASUREMENT}

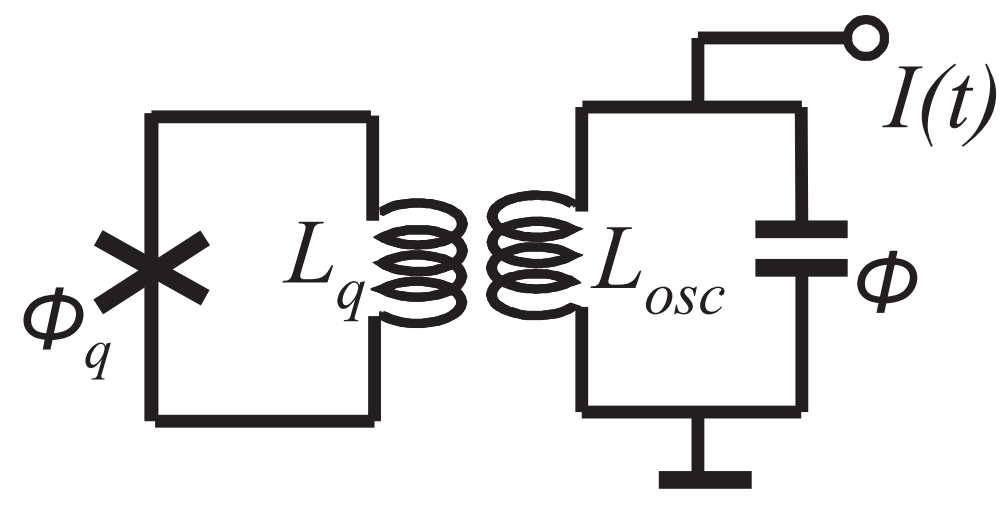

FIG. 7: Single-contact flux qubit inductively coupled to a linear oscillator.

Consider first a simpler circuit with a linear LC-oscillator replacing the dc SQUID (Fig. 7). Such a device, rf-SQUID inductively coupled to a linear oscillator is a classical device employed for many years for precise measurement of magnetic field ${ }^{57}$. The principle of operation is based on the magnetic field dependence of the Josephson inductance, which affects the resonance frequency of the oscillator probed by an external rf signal. The classical Hamiltonian for this circuit, again assuming for simplicity a single Josephson junction in the qubit loop, has the form,

$$
H=E_{C q} n_{q}^{2}-E_{J q} \cos \phi_{q}+E_{L q} \tilde{\phi}_{q}^{2}+E_{M} \tilde{\phi}_{q} \phi+E_{C o s c} n^{2}+E_{L o s c} \phi^{2}+\frac{\hbar}{2 e} I(t) \phi .
$$

Neglecting the junction capacitance energy, and expanding the qubit potential energy near the minimum point $\tilde{\phi}_{q 0}\left(f_{q}\right)$,

$$
-E_{J q} \cos \phi_{q}+E_{L q} \tilde{\phi}_{q}^{2} \equiv U\left(\tilde{\phi}_{q}\right)=U\left(\tilde{\phi}_{q 0}\right)+\frac{1}{2} U^{\prime \prime}\left(\tilde{\phi}_{q 0}\right)\left(\tilde{\phi}_{q}-\tilde{\phi}_{q 0}\right)^{2}
$$

we define an effective inductance of the qubit circuit $L_{q}^{e f f}$ via the relation

$$
\frac{1}{2} U^{\prime \prime}\left(\tilde{\phi}_{q 0}\right)=\left(\Phi_{0} / 2 \pi\right)^{2} / 2 L_{q}^{e f f}\left(f_{q}\right)=E_{L q}^{e f f}\left(f_{q}\right)
$$

After having diagonalized the total potential energy, we obtain the shift of the oscillator inductive energy due to coupling to the qubit,

$$
\tilde{E}_{L o s c}=E_{L o s c}-\frac{E_{M}^{2}}{4 E_{L q}^{e f f}\left(f_{q}\right)} .
$$


This gives rise to a shift of the oscillator resonance frequency depending on the magnetic flux through the qubit loop, which is probed with an external rf signal $I(t)$.

A similar measurement procedure also applies to the quantum regime. The quantum Hamiltonian for the same circuit has the form, taking into account Eqs. (41) and (42),

$$
H=-\frac{1}{2}\left(\epsilon \sigma_{z}+\Delta \sigma_{x}\right)+E_{M} \phi_{0} \phi \sigma_{z}+E_{C o s c} n^{2}+E_{L o s c} \phi^{2}+\frac{\hbar}{2 e} I(t) \phi .
$$

For a slow oscillator and weak coupling, the Hamiltonian can be rotated to a qubit eigenbasis, and expanded with respect to the coupling term,

$$
H=-\frac{1}{2} \varepsilon \sigma_{z}-\frac{\left(E_{M} \phi_{0}\right)^{2}}{\varepsilon} \phi^{2} \sigma_{z}+E_{C o s c} n^{2}+E_{L o s c} \phi^{2}+\frac{\hbar}{2 e} I(t) \phi
$$

(here the term linear in $\phi$ is omitted since it only produces a non-essential small shift of the oscillator coordinate; $\varepsilon=\sqrt{\epsilon^{2}+\Delta^{2}}$ ). The second term in Eq. (53) provides the shift of the oscillator inductive energy depending on the qubit state,

$$
\tilde{E}_{L o s c}=E_{L o s c}-\frac{E_{M}^{2} \phi_{0}^{2}}{\varepsilon} \sigma_{z}
$$

Comparing this quantum result with the classical equation (51), we are able to identify the quantum inductance of the qubit,

$$
L_{Q}=\left(\frac{2 \pi}{\Phi_{0}}\right)^{2} \frac{2 \phi_{0}^{2}}{\varepsilon} \sigma_{z}
$$

The quantum inductance is inversely proportional to the qubit level splitting, similar to the quantum capacitance of charge qubits, and it approaches its maximum value at the degeneracy point.

This conclusion also applies to the 3 -junction flux qubit ${ }^{32}$; the only difference is due to the suppressed coupling of the qubit to the outside world discussed in the previous section below Eq. (46). In this case, an additional small factor, $E_{J q} / E_{L q}$, appears in the the coupling term in Eq. (52), giving rise to the following equation for the quantum inductance of the 3 -junction qubit,

$$
L_{Q}=\left(\frac{2 \pi}{\Phi_{0}}\right)^{2}\left(\frac{E_{J q}}{E_{L q}}\right)^{2} \frac{2 \phi_{0}^{2}}{\varepsilon} \sigma_{z}
$$

Proceeding with the case of the readout dc SQUID, Eq. (46), we find that this case seems to be qualitatively different from the LC-oscillator readout: the qubit-meter coupling is non-linear. Obviously, this results from the fact that the qubit is not directly coupled to the readout $\phi_{-}$-oscillator, but rather via an intermediate $\phi_{s}$-oscillator, the elimination of which results in the non-linear coupling.

One way to solve the problem is to displace the $\phi_{-}$-oscillator by applying a constant current bias, $I(t)=I_{0}+I_{1}(t)$. Then expanding the potential energy around the minimum point, $\phi_{-}=\bar{\phi}_{-}+\theta$, where $\bar{\phi}_{-}$satisfies the equation, $2 E_{J s} \cos \left(f_{s} / 2\right) \sin \bar{\phi}_{-}=-(\hbar / 2 e) I_{0}$, we arrive, in the linear approximation, at a Hamiltonian similar to Eq. (52),

$$
\begin{gathered}
H=-\frac{1}{2}\left(\epsilon \sigma_{z}+\Delta \sigma_{x}\right)+\frac{1}{2} E_{C s} n_{-}^{2}+E_{J s} \cos \bar{\phi}_{-} \cos \left(f_{s} / 2\right) \theta^{2}+\tilde{\lambda} E_{J s} \theta \sigma_{z}+\frac{\hbar}{2 e} I_{1}(t) \theta \\
\tilde{\lambda}=\lambda \frac{\hbar I_{0} E_{J q}}{2 e E_{L q}} \tan \left(f_{s} / 2\right) .
\end{gathered}
$$

Another solution would be to access directly the $\phi_{s}$-oscillator linearly coupled to the qubit. This can be done, for example, by driving a bias flux through the SQUID, $f_{s}(t)$. For small variation of phases in Eq. (43), the oscillators decouple, and the relevant part of the Hamiltonian, taking into account Eqs. (41) and (42), approaches a form similar to Eq. (52),

$$
H=-\frac{1}{2}\left(\epsilon \sigma_{z}+\Delta \sigma_{x}\right)++2 E_{C s} n_{s}^{2}+E_{J s} \phi_{s}^{2}+\frac{E_{M} E_{J q} \phi_{0}}{E_{L q}} \phi_{s} \sigma_{z}+2 E_{J s} f_{s}(t) \phi_{s} .
$$


Finally, the $\phi_{s}$-oscillator may be accessed by using an asymmetric SQUID, with different inductances of the left and right legs (Fig. 1), $L_{1} \neq L_{2}, L_{1}+L_{2}=L_{s}$. In this case, the coupling of the SQUID to an external current source in Eq. (39) becomes modified to

$$
\frac{\hbar}{4 e} I(t)\left(\frac{L_{2}}{L_{s}} \phi_{1}-\frac{L_{1}}{L_{s}} \phi_{2}\right)
$$

which results in direct coupling of the probing current to the $\phi_{s}$-oscillator. At zero flux bias, $f_{s}=0$, the two $\phi_{ \pm}$-oscillators do not interact, and the relevant part of the Hamiltonian reads,

$$
H=-\frac{1}{2}\left(\epsilon \sigma_{z}+\Delta \sigma_{x}\right)+2 E_{C s} n_{s}^{2}+\left(E_{L s}+E_{J s}\right) \phi_{s}^{2}+\frac{E_{M} E_{J q} \phi_{0}}{E_{L q}} \phi_{s} \sigma_{z}+\frac{\hbar}{2 e} I(t) \frac{L_{2}-L_{1}}{L_{s}} \phi_{s} .
$$

Thus there are several ways to employ the dc SQUID for dispersive measurement of the qubit quantum inductance. Note, however, that in the latter case the inductive energy of the SQUID plays a role, and for small SQUID inductance $\left(E_{L s} \gg E_{J s}\right)$ the oscillator frequency may become large, violating the adiabatic regime assumed in the derivation.

\section{CONCLUDING REMARKS}

In this paper we have outlined some practical schemes for capacitive and inductive readout, detecting the state of a qubit by reflecting microwaves from an oscillator circuit, the phase shift measuring the changes in charge or magnetic flux induced by a qubit, allowing to distinguish between the different states $|0\rangle$ and $|1\rangle$ of the qubit. In particular we focussed attention on the single-Cooper-pair box (SCB), the effective capacitance of which can be defined as the derivative of the induced charge with respect to gate voltage. In addition to the geometric capacitance, there is the quantum capacitance due to the level dispersion at the anti-crossing caused by the Josephson coupling. We described the process of reflection of quantized radiation and derived expressions for the shortest measurement time needed to resolve the qubit states, suggesting that a low Q should be advantageous for weak back action fast readout.

\section{ACKNOWLEDGMENT}

This work was supported in part by the European Commission through projects FP5-39083-SQUBIT-2 and FP6015708 EuroSQIP of the IST Priority (Disclaimer: www.eurosqip.org), and by the Swedish Research Council. 
1 D. Esteve and D. Vion: "Solid state quantum bit circuits", Les Houches Summer School-Session LXXXI on Nanoscopic Quantum Physics, (2004).

2 J.M. Elzerman, R. Hanson, L.H. Willems van Beveren, B. Witkamp, J.S. Greidanus, R.N. Schouten, S. De Franceschi, S. Tarucha, L.M.K. Vandersypen and L.P. Kouwenhoven: "Semiconductore few-electron quantum dots as spin qubits", in Quantum Dots: A Doorway to Nanoscle Physics, Lecture Notes in Physics Vol. 667, ed. W.D. Heiss, (2005).

3 G. Wendin and V.S. Shumeiko: "Superconducting circuits, qubits and computing", Handbook of Theoretical and Computational Nanotechnology, Eds. M. Rieth and W. Schommers. Ch. 12,, American Scientific Publishers, 2006; cond-mat/0508729

${ }^{4}$ C.F. Roos, G.P.T. Lancaster, M. Riebe, H. Häffner, W. Hänsel, S. Gulde, C. Becher, J. Eschner, F. Schmidt-Kaler and R. Blatt: "Bell states of atoms with ultralong lifetimes and their tomographic state analysis", Phys. Rev. Lett. 92, 220402 (2004).

5 M. Riebe, H.Häffner, C.F. Roos, W.Hänsel, J. Benhelm, G.P.T. Lancaster, T.W. Körber, C. Becher, F. Schmidt-Kaler, D.F.V. James and R. Blatt: "Deterministic quantum teleportation with atoms", Nature 429, 734 (2004).

${ }^{6}$ M.D. Barrett, J. Chiaverini, T. Schaetz, J. Britton, W.M. Itano, J. D. Jost, E. Knill, C. Langer, D. Leibfried, R. Ozeri and D.J. Wineland: "Deterministic quantum teleportation of atomic qubits", Nature 429, 737 (2004).

7 J. Chiaverini, D. Leibfried, T. Schaetz, M.D. Barrett, R.B. Blakestad, J. Britton, W.M. Itano, J. D. Jost, E. Knill, C. Langer, R. Ozeri and D.J. Wineland: "Realization of quantum error correction", Nature 432, 602 (2004).

8 D. Leibfried, E. Knill, S. Seidelin, J. Britton, R. B. Blakestad, J. Chiaverini, D. B. Hume, W. M. Itano, J. D. Jost, C. Langer, R. Ozeri, R. Reichle, and D. J. Wineland: "Creation of a six-atom 'Schrdinger cat' state", Nature 438, 639 (2005).

9 H. Häffner, W. Hänsel, C. F. Roos, J. Benhelm, D. Chek-al-kar, M. Chwalla, T. Körber, U. D. Rapol, M. Riebe, P. O. Schmidt, C. Becher, O. Gühne, W. Dür, and R. Blatt: "Scalable multiparticle entanglement of trapped ions", Nature 438, $643(2005)$.

10 A. Blais, R.-S. Huang, A. Wallraff, S. M. Girvin R. J. Schoelkopf: "Cavity quantum electrodynamics for superconducting electrical circuits: an architecture for quantum computation", Phys. Rev. A 69, 062320 (2004).

11 I. Rau, G. Johansson, and A. Shnirman: "Cavity QED in superconducting circuits: susceptibility at elevated temperatures", Phys. Rev. B 70, 054521 (2004).

12 A. Wallraff, D. Schuster, A. Blais, L. Frunzo, R.-S. Huang, J. Majer, S. Kumar, S.M. Girvin and R. J. Schoelkopf: "Cavity quantum electrodynamics: Coherent coupling of a single photon to a Cooper pair box", Nature 431, 165 (2004).

13 Quantum Information Processing \& Communications Fet Proactive Initiative in the 6th Framework Programme Strategic Report; http://www.cordis.lu/ist/fet/qipc.htm

14 ARDA Quantum Computation Roadmap; http://qist.lanl.gov/

15 M. A. Sillanpää, T. Lehtinen, A. Paila, Yu. Makhlin, L. Roschier, and P. J. Hakonen Phys. Rev. Lett. 95, 206806 (2005)

16 T. Duty, G. Johansson, K. Bladh, D. Gunnarsson, C. Wilson and P. Delsing: "Observation of quantum capacitance in the Cooper-pair transistor", Phys. Rev. Lett. 95, 206807 (2005).

17 A. Zorin: "Cooper pair qubit and electrometer in one device", Physica C 368, 284 (2002).

18 E. Il'ichev, N. Oukhanski, A. Izmalkov, Th. Wagner, M. Grajcar, H.-G. Meyer, A.Yu. Smirnov, Alec Maassen van den Brink, M.H.S. Amin and A.M. Zagoskin: "Continuous monitoring of Rabi oscillations in a Josephson flux qubit", Phys. Rev. Lett. 91, 097906 (2003).

19 M. Sillanpää, L. Roschier, and P. Hakonen: "The Inductive Single-Electron Transistor (L-SET)", Phys. Rev. Lett. 93, 066805 (2004).

20 A. Lupascu, C. J. P. M. Harmans and J. E. Mooij: "State detection of a superconducting flux qubit using a dc-SQUID in the inductive mode", Phys. Rev. B 71, 184506 (2005).

21 P. Bertet, I. Chiorescu, C.J.P.M. Harmans and J.E. Mooij: "Dephasing of a flux qubit coupled to a harmonic oscillator", (2005); cond-mat/0507290

22 Yu. Makhlin, G. Schön, and A. Shnirman: "Quantum state engineering with Josephson-junction devices", Rev. Mod. Phys. 73, 357 (2001).

23 F.K. Wilhelm: "An asymptotical von-Neumann measurement strategy for solid-state quantum bits", Phys. Rev. 68, 060503(R) (2003).

${ }^{24}$ Y. Nakamura, Yu. Pashkin and J.S. Tsai: "Coherent control of macroscopic quantum states in a single-Cooper-pair box", Nature 398, 786 (1999).

${ }^{25}$ T. Duty, D. Gunnarsson, K. Bladh and P. Delsing: "Coherent dynamics of a charge qubit", Phys. Rev. B 69, 1405023(R) (2004).

26 R.J. Schoelkopf, P. Wahlgren, A.A. Kozhevnikov, P. Delsing and D.E. Prober: "The radio-frequency single-electron transistor (rf-SET): A fast and ultra-sensititive electrometer", Science 280, 1238 (1998).

27 O. Astafiev, Yu. A. Pashkin, T. Yamamoto, Y. Nakamura, and J. S. Tsai: "Single-shot measurement of the Josephson charge qubit", Phys. Rev. B 69, 180507(R) (2004).

28 D. Vion, A. Cottet, A. Aassime, P. Joyez, H. Pothier, C. Urbina, D. Esteve and M.H. Devoret: "Manipulating the quantum state of an electrical circuit", Science 296, 886 (2002).

29 D. Vion, A. Aassime, A. Cottet, P. Joyez, H. Pothier, C. Urbina, D. Esteve and M.H. Devoret: "Rabi oscillations, Ramsey fringes and spin echoes in an electrical circuit", Fortschritte der Physik 51, 462 (2003).

30 S. Corlevi, W. Guichard, F.W.J. Hekking and D.B. Haviland: "Phase-Charge Duality of a Josephson junction in a fluctuating electromagnetic environment", (2005); cond-mat/0510504 
31 J. Sjostrand, J. Walter, E. Tholen, H. Hansson, D. Haviland and A. Karlhede: "Phase space topology of a switching current detector", (2005); cond-mat/0510246

32 J.E. Mooij, T.P. Orlando, L. Levitov, Lin Tian, C.H. van der Wal, and S. Lloyd: "Josephson persistent current qubit", Science 285, 1036 (1999).

33 C.H. van der Wal, A.C.J. ter Haar, F. Wilhelm, R.N. Schouten, C.J.P.M. Harmans, T.P. Orlando, S. Lloyd J.E. and Mooij: "Quantum superposition of macroscopic persistent-current states", Science 290, 773 (2000).

34 J.R. Friedman, V. Patel, W. Chen, S.K. Tolpygo and J.E. Lukens: "Detection of a Schrödinger's cat state in an rf-SQUID", Nature 406, 43 (2000).

${ }^{35}$ I. Chiorescu, Y. Nakamura, C.J.P.M. Harmans, J.E. Mooij: "Coherent Quantum Dynamics of a Superconducting FluxQubit", Science 299, 1869 (2003).

${ }^{36}$ I. Chiorescu, P. Bertet, K. Semba, Y. Nakamura, C.J.P.M. Harmans and J.E. Mooij: "Coherent dynamics of a flux qubit coupled to a harmonic oscillator", Nature 431, 159 (2004).

37 A. Aassime, D.Gunnarsson, K. Bladh, P. Delsing, and R. Schoelkopf: "Radio-frequency single-electron transistor: Toward the shot-noise limit", Appl. Phys. Lett. 79, 4031 (2001)

38 A. Aassime, G. Johansson, G. Wendin, P. Delsing, and R. Schoelkopf: "Radio-frequency single-electron transistor as read-out device for qubits: Charge sensitivity and back-action", Phys. Rev. Lett. 86, 3376 (2001)

39 G. Johansson, A. Käck and G. Wendin: "Single Shot Charge Qubit read-out using a Single Electron Transistor: Back-action and Fidelity", Phys. Rev. Lett. 88, 046802 (2002)

40 A. Käck, G. Wendin, and G. Johansson: "Full frequency voltage noise spectral density of a single electron transistor", Phys. Rev. B 67, 035301 (2003)

41 O. Astafiev, Yu. A. Pashkin, Y. Nakamura, T. Yamamoto, and J. S. Tsai: "Quantum Noise in the Josephson Charge Qubit", Phys. Rev. Lett. 93, 267007 (2004).

42 G. Ithier, E. Collin, P. Joyez, P. J. Meeson, D. Vion, D. Esteve, F. Chiarello, A. Shnirman, Y. Makhlin, J. Schriefl, and G. Schön: "Decoherence in a superconducting quantum bit circuit ", Phys. Rev. B 72, 134519 (2005)

43 M. Büttiker,: "Zero-current persistent potential drop across small-capacitance Josephson junctions", Phys. Rev. B 36, 3548 (1987)

44 V. Bouchiat, D. Vion, P. Joyez, D. Esteve, and M. H. Devoret: "Quantum Coherence with a Single Cooper Pair", Phys. Scr. T76, 165 (1998).

45 A. Widom, G. Megaloudis, T. D. Clark, J. E. Mutton, R. J. Prance and H. Prance: "The Josephson pendulum as a nonlinear capacitor", J. Low Temp. Phys. 57, 651 (1984)

46 D. V. Averin, A. B. Zorin, and K. K. Likharev: "Bloch oscillations in small Josephson junctions", Sov. Phys. JETP 61, 407 (1985)

47 K. K. Likharev and A. B. Zorin: "Theory of the Bloch-wave oscillations in small Josephson junctions", J. Low Temp. Phys. 59, 347 (1985)

48 G. Johansson, L. Tornberg, and C. Wilson: "Quantum efficient charge qubit read-out", submitted to Phys. Rev. Letters; condmat/06xxx.

49 B. Yurke and J. S. Denker: "Quantum network theory", Phys. Rev. A 29, 1419-1437 (1984)

50 M. Devoret: "Quantum fluctuations in electrical circuits", Quantum Fluctuations, Eds. S. Reynaud, E. Giacobino and J. Zinn-Justin, Les Houches, Session LXIII, 1995, Elsevier Science B.V., 1997.

51 S. M. Barnett, P. M. Radmore, Methods in Theoretical Quantum Optics Oxford University Press Inc. New York 1997

52 C. W. Gardiner, P. Zoller, Quantum Noise, A Handbook of Markovian and Non-Markovian Quantum Stochastic Methods with Applications to Quantum Optics (Springer-Verlag Berlin Heidelberg, 1991 ,2000)

53 A. Wallraff, D. I. Schuster, A. Blais, L. Frunzio, J. Majer, M. H. Devoret, S. M. Girvin, and R. J. Schoelkopf: "Approaching Unit Visibility for Control of a Superconducting Qubit with Dispersive Readout", Phys. Rev. Lett. 95, 060501 (2005).

${ }^{54}$ G. Burkard: "Theory of solid state quantum information processing", prepared for Handbook of Theoretical and Computational Nanotechnology (2004); cond-mat/0409626

55 H. Takayanagi, H. Tanaka, S. Saito and H. Nakano, Readout of the qubit state with a dc-SQUID, Superlatt. and Microstructures 32, 221 (2003).

${ }^{56}$ M. Staf, "Quantum mechanics of superconducting circuits", Master Thesis, Göteborg, 2003.

57 A. Barone and G. Paterno, Physics and Applications of the Josephson Effect, (Wiley, New York), 1982. 